Physical-

s.

\title{
The Assessment of Preschool Children's Participation: Internal Consistency and Construct Validity
}

\section{Mary Law, Gillian King, Theresa Petrenchik, Marilyn Kertoy \& Dana Anaby}

To cite this article: Mary Law, Gillian King, Theresa Petrenchik, Marilyn Kertoy \& Dana Anaby (2012) The Assessment of Preschool Children's Participation: Internal Consistency and Construct Validity, Physical \& Occupational Therapy In Pediatrics, 32:3, 272-287, DOI: 10.3109/01942638.2012.662584

To link to this article: https://doi.org/10.3109/01942638.2012.662584

曲 Published online: 05 Mar 2012.

Submit your article to this journal $2 \pi$

Lll Article views: 1628

View related articles $\square$

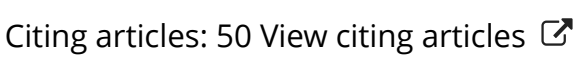




\title{
The Assessment of Preschool Children's Participation: Internal Consistency and Construct Validity
}

\author{
Mary Law ${ }^{1}$, Gillian King ${ }^{2}$, Theresa Petrenchik ${ }^{1}$, Marilyn Kertoy ${ }^{3}, \&$ \\ Dana Anaby ${ }^{4}$
}

${ }^{1}$ CanChild Centre for Childhood Disability Research and School of Rehabilitation Science, McMaster University, Hamilton, Ontario, Canada, ${ }^{2}$ Bloorview Research Institute, Toronto, Ontario, Canada, ${ }^{3}$ School of Communication Sciences and Disorders, University of Western Ontario, London, Ontario, Canada, ${ }^{4}$ School of Physical and Occupational Therapy, McGill University, Montreal, Quebec, Canada

\begin{abstract}
Participation in activities provides the means for young children to learn, play, develop skills, and develop a sense of personal identity. The Assessment of Preschool Children's Participation (APCP) is a newly developed measure to capture the participation of children aged 2 to 5 years and 11 months in the areas of play, skill development, active physical recreation, and social activities. Data from a clinical trial involving 120 children with cerebral palsy indicated that the APCP has moderate to very good internal consistency. The measure distinguishes between children below or above 4 years of age across levels of the Gross Motor Classification System, and between income levels below or above the median regional income range. The APCP, with a focus on preschool children, has potential use for assessment and identification of activity areas in which the child is participating and areas in which participation may be restricted.
\end{abstract}

KEYWORDS. Cerebral palsy, leisure and recreation, play, playfulness, psychometric testing

Participation is defined by the World Health Organization (WHO) as "involvement in a life situation" (World Health Organization, 2001, p. 10). In addition, Coster and Khetani (2008, p. 643) suggested that life situations are "sets of organized sequences of activities directed toward a personally or socially meaningful goal." Participation in activities is the context in which young children learn, form friendships, develop skills and competencies, express creativity, and develop a sense of personal identity (Coatsworth, Palen, \& Sharp, 2006).

Address correspondence to: Mary Law, PhD, CanChild Centre for Childhood Disability Research and School of Rehabilitation Science, McMaster University, IAHS Bldg., 1400 Main St W., Hamilton, Ontario, Canada L8S1C7 (E-mail: lawm@mcmaster.ca). 
A majority of the recent research regarding the participation of children with disabilities has focused on children over the age of 6 years. There has been little research about the participation of preschool children, particularly research focusing on the measurement of sets of activities. A challenge in studying participation for children under 6 years of age is the lack of validated measures of the participation for preschool children. Such measures have potential use to identify patterns of participation for activities for both families and service providers.

The Assessment of Preschool Children's Participation (APCP) (King, Law, Petrenchik, \& Kertoy, 2006c) is a newly developed measure designed to capture the activity participation of children aged 2 to 5 years and 11 months. The purpose of this paper is to provide data regarding psychometric properties of the APCP among children with cerebral palsy. More specifically, we examined the internal consistency and construct validity of the APCP by conducting an item analysis and testing group differences in participation using variables that are known to be related to the construct of childhood participation. To further establish validity, we examined the association between number of health conditions in addition to cerebral palsy with levels of participation diversity and intensity across all activity types/domains.

\section{BACKGROUND INFORMATION}

The participation of children in everyday activities is associated with the cultivation of children's social relationships, the development of essential skills and competencies (Larson \& Verma, 1999; Mahoney, Larson, \& Eccles, 2005), and the promotion of mental, physical, and behavioral health (Sandler, Ayers, Suter, Schultz, \& Twohey-Jacobs, 2004; Wright \& Masten, 2005). The preschool years are particularly important for development across all areas, as development is impacted significantly by a child's participation in everyday social and solitary activities.

Dunst, Hamby, Trivette, \& Raab (2002) examined participation in family and community activities of preschool children from birth to 6 years who had or were at risk for a developmental delay. Using family and community surveys with over 1,400 parents, they found that children participated in a large range of activities, with considerable variability in individual participation patterns. The percentage of children participating in different types of activities increased in a linear fashion as children got older.

The participation of preschool children with disabilities has not been studied in depth, as most research has focused on measurement of body function and structure (e.g., reaching, range of motion, eye-hand coordination) or activity limitations (e.g., ability to hold a spoon independently, putting on clothes, or undo buttons). Simeonsson et al. (2003) wrote about the need to develop and test measures that reflect the broad content of the new International Classification of Functioning, Disability and Health (ICF) (WHO, 2001). They identified 14 measures commonly used in pediatric rehabilitation, the majority of which focused on assessment of functional and developmental areas rather than participation. Over the past decade, there has been substantial development of health-related quality-of-life measures for children, but less development of measures focused specifically on the participation domain of the ICF. As well, there are several assessments of preschool play skills, which include only one area of participation such as outdoor play. 
The Lifestyle Assessment Questionnaire (LAQ) (Jessen, Colver, Mackie, \& Jarvis, 2003) is an assessment designed to measure the impact of disability across the participation domains of communication, mobility, self-care, domestic life, social and civic activities, and family impact. The LAQ has been used to assess impact of disability in two studies of children 5 years of age (Forsyth, Colver, Alvanides, Woolley, \& Lowe, 2007; Welsh, Jarvis, Hammal \& Colver, 2006), but is predominantly used for assessment in research with children aged over 5 years. The Functional Independence Measure for Children (Wee-FIM) (Msall et al., 1994) and the Pediatric Evaluation of Disability Inventory (PEDI) (Haley, Coster, Ludlow, Haltiwanger, \& Andrellos, 1992) are the most commonly used assessments in preschool intervention studies for children with rehabilitation needs. Both of these assessments focus on the level of performance and include items related to mobility, selfcare, and social functioning, but do not include broader sets of activities that comprise preschool participation such as play and community activities.

One preschool participation assessment, which has been developed and is currently undergoing psychometric testing, is the Preschool Activity Card Sort (PACS) (Berg \& LaVesser, 2006; Stoffel \& Berg, 2008). The PACS is designed to be used to set intervention goals for children 3 to 6 years of age. The PACS covers six domains using 85 activity cards. The assessment uses photographs of childhood activities across the domains of self-care, community mobility, leisure, social interaction, domestic chores, and education. Through this assessment, parents use the activity photographs to identify the activities in which their child participates and five activities for which they wish occupational therapy services. The PACS has been translated and tested in Spanish (Stoffel \& Berg, 2008) and the English version demonstrated that it discriminated between preschool children with and without autism (LaVesser \& Berg, 2011).

The APCP is a measure designed to document children's participation in day-today activities. The APCP is modeled after the Children's Assessment of Participation and Enjoyment (CAPE) (King et al., 2004), which uses drawings of everyday activities to ask parents and children about the nature and frequency of participation in recreational, active physical, social, skill-based, and self-improvement activities during non-school hours. The APCP includes 45 drawings of everyday activities in the areas of play, skill development, active physical recreation, and social activities. Parents are asked to identify activities their child has participated in during the past 4 months and how often they did them. In developing the APCP, we recognized that the participation of preschool children is significantly influenced by their family and the participation choices that parents make. While preschool children can make choices about activities, these choices are based on what is available to them in settings typically structured by adults. Thus, the participation of preschool children is often a reflection of their family's participation choices, child-care needs, and opportunities available within the child's immediate environment.

Findings from previous research on childhood participation guided the development of hypotheses regarding construct validity of the APCP. In a sample of preschool children without disabilities, researchers found evidence of age-related differences in participation across groups of children at risk or with a developmental delay (Dunst et al., 2002). Studies of preschool children with cerebral palsy have found several predictors of participation, including severity of impairment (Forsyth 
et al., 2007), the level of cognitive, motor, and communicative functioning, and families' district of residence (Hammal, Jarvis \& Colver, 2004). Among children aged 6 years and older with a disability, participation differences have been found to be significantly influenced by a child's age (Law et al., 2006), sex (King, Law, Hurley, Petrenchik, \& Schwellnus, 2010), functional ability (King et al., 2006a; Majnemer et al., 2010; Palisano et al., 2009, level of family income (Law et al., 2006), child preferences (King et al., 2006a), and family's participation in social and recreational activities (King et al., 2006a). Children with disabilities generally participate in lower levels of recreation and leisure activities than children without disabilities (Bult et al., 2010; Imms, Reilly, Carlin, \& Dodd, 2008; King et al., 2010; Law et al., 2006; Majnemer et al., 2008).

The purpose of this paper is to provide an evaluation of psychometric properties of the APCP with respect to its ability to discriminate patterns of intensity and diversity of participation as a function of children's age, sex, level of gross motor function, and annual family income. Data presented in this paper were collected through a randomized controlled trial evaluating the efficacy of a child-focused versus context-focused intervention in improving performance of functional tasks and mobility in young children with cerebral palsy (Law et al., 2011). The trial included 128 children with cerebral palsy across Levels I-V on the Gross Motor Classification System (GMFCS) (Palisano et al., 1997). Children received child-focused $(N=$ $71)$ or context-focused intervention $(N=57)$ over 6 months, returning to their regular therapy schedule and approach between the end of intervention at 6 months and follow-up at 9 months. The primary outcome was performance on the PEDI (Haley et al, 1992). Outcome evaluators were masked to group assignment and completed assessments at baseline, 6 months, and 9 months. The analyses in this paper used the baseline data for children in the trial who were 2 to 5 years and 11 months of age $(N=120)$. The analyses were designed to investigate the following hypotheses regarding the construct validity of the APCP:

1. Children over 4 years of age will be involved in more activities than younger children, but not more frequently.

2. Girls will participate in more social activities than boys; boys will participate in more active physical recreation activities, and more frequently.

3. Children with higher levels of impairments in self-care and motor functioning or higher number of health conditions will participate in fewer activities and with less frequency than children with lower levels.

4. Children living in families with higher median annual incomes will participate in more activities and with greater frequency than children living in lower income families.

\section{Participants}

Participants were 120 children with cerebral palsy who were between 2 years and 5 years and 11 months of age and attended one of 19 children's rehabilitation centers in Ontario and Alberta, Canada. Ethics approval for the study was obtained from the Office of Research Ethics at McMaster University and the University of Alberta, and parents provided informed consent for the study. The sample included 
TABLE 1. Characteristics of 120 Children with Cerebral Palsy

\begin{tabular}{|c|c|c|c|}
\hline Variable & Category & $N$ & $\%$ \\
\hline \multirow[t]{2}{*}{ Sex } & Boys & 71 & 59.2 \\
\hline & Girls & 49 & 40.8 \\
\hline \multirow[t]{5}{*}{ GMFCS } & Level I & 36 & 30 \\
\hline & Level II & 21 & 17.5 \\
\hline & Level III & 18 & 15 \\
\hline & Level IV & 21 & 17.5 \\
\hline & Level V & 24 & 20 \\
\hline \multirow[t]{8}{*}{ Family income } & $<\$ 15,000$ & 7 & 6.7 \\
\hline & $\$ 15,000-\$ 29,999$ & 11 & 9.2 \\
\hline & $\$ 30,000-\$ 44,999$ & 14 & 11.7 \\
\hline & $\$ 45,000-\$ 59,999$ & 15 & 12.5 \\
\hline & $\$ 60,000-\$ 74,999$ & 13 & 10.8 \\
\hline & $\$ 75,000-\$ 89,999$ & 18 & 15 \\
\hline & $\geq \$ 90,000$ & 34 & 28.3 \\
\hline & Missing & 7 & 5.8 \\
\hline \multirow[t]{3}{*}{ Family unit } & Two-parent family & 106 & 88.3 \\
\hline & Single parent family & 13 & 10.8 \\
\hline & Missing & 1 & 0.8 \\
\hline \multirow[t]{6}{*}{ Ethnicity background } & Caucasian & 92 & 76.7 \\
\hline & Asian & 9 & 7.5 \\
\hline & African-American & 6 & 5 \\
\hline & Latin & 4 & 3.3 \\
\hline & N. American Indian & 3 & 2.5 \\
\hline & Others & 6 & 5 \\
\hline \multirow[t]{5}{*}{ Community } & Large metropolitan & 23 & 19.2 \\
\hline & Medium metropolitan & 38 & 31.7 \\
\hline & Small-metro & 25 & 20.8 \\
\hline & Non-metro urbanized area & 19 & 15.8 \\
\hline & Rural & 15 & 12.5 \\
\hline \multirow[t]{7}{*}{ Additional health conditions } & Behavioral disorder & 3 & 2.5 \\
\hline & Developmental delay & 61 & 50.8 \\
\hline & Seizure disorder & 30 & 25 \\
\hline & Vision impairment & 42 & 35 \\
\hline & Hearing impairment & 9 & 7.5 \\
\hline & Learning disorder & 15 & 12.5 \\
\hline & Others & 33 & 27.5 \\
\hline
\end{tabular}

120 children aged 2 to 6 years (mean $=4.1, \mathrm{SD}=1.1$ ), all diagnosed with cerebral palsy. The number of additional health conditions ranged from 0 to 6 (mean $=1.3$, $\mathrm{SD}=1.3$, median $=1$ ). Table 1 summarizes additional demographic and clinical information for the children in the study and their families.

\section{Measures}

The evaluation of psychometric properties of the APCP used data from several outcome measures. The PEDI (Haley et al., 1992) was used to measure the performance of functional tasks of self-care and mobility. Each domain, i.e., self-care and mobility, is measured using a Functional Skills Scale (FSS) that is rated as 0 (unable, or limited capacity to perform the skill) or 1 (able to perform the skill), and a Caregiver Assistance Scale (CAS) that is rated from 0 (total assistance) to 5 (independent). Thus, four scores are generated from the PEDI. The PEDI has been 
validated in many studies and has excellent reliability and validity for this study population.

The GMFCS (Palisano et al., 1997) is a five-level system that classifies children's gross motor function in everyday routines within four age strata. Specific levels on the GMFCS are based on the child's motor abilities and use of mobility devices.

\section{APCP Development}

The APCP is a paper and pencil, parent completed questionnaire containing 45 drawings of everyday activities. Parents are asked to identify activities their child has participated in during the past 4 months and how often they did them, as done in the CAPE (King et al., 2006b). The purpose of the assessment is to capture preschool children's activity patterns in the areas of play (e.g., playing with toys, creating a craft), skill development (e.g., drawing and coloring, taking music lessons), active physical recreation (e.g., riding a tricycle, playing on playground equipment), and social activities (e.g., playing a board game, going on an outing). The APCP reflects the conceptual framework underlying WHO's (2001) ICF. This framework distinguishes between impairments, activity limitations, and participation restrictions. The APCP focuses on a subset of the ICF domains of participation. The items constitute a categorization of sets of activities based on a comprehensive review of the literature and feedback from families through a pilot study, as well as items from the CAPE (i.e., skill-based, physical, and social) and scoring scales deemed appropriate for younger children. The APCP includes 7 items not in the CAPE, 23 identical items, and 15 similar items modified to fit preschool children.

Like the CAPE, the APCP captures aspects of children's participation in a range of non-academic activities in a variety of everyday environments, including home, preschool, nursery school, or kindergarten and child care arrangements such as day care, before and after school programs, and supervised care. Unlike the CAPE, the APCP is parent-completed (rather than child- or youth-completed) and does not include measurements of where and with whom activities take place, or the child's rating of enjoyment. The items selected for use in the APCP reflect the most common types of everyday activities discussed in the literature across populations of children and age groups. Item generation was based on review of developmental literature and existing measures of participation, which excluded basic self-care activities.

The initial version of the APCP included 43 items; initial pilot testing identified additional five new items, for a total of 48 items. As previously indicated, items are organized into four activity areas, three of which are in the CAPE - play activities, skill development, active physical recreation, and social activities. On the assessment, parents identify "yes" or "no" to indicate whether their child participates in each activity. If the child does participate, they record how often the child participated over the past 4 months using a 7-point ordinal scale ranging from once over the past 4 months to once daily or more. Two scores are generated for each item and across the four activity areas. Participation diversity is a count of the total number of a child's reported activities over the previous 4 months. For group data, diversity can also be reported as a percentage of activities overall and by activity type. Participation intensity represents the average amount of time that a child spends participating in activities across the total number of possible activities. Intensity 
is calculated by dividing the sum of frequency across all items by the number of possible items in each activity area. Preliminary testing with 57 parents of children with typical development attending four local day care centers in southern Ontario, Canada indicated that the activity scales had levels of internal consistency between 0.60 and 0.70 ; these levels improved after the elimination of three items that were endorsed less than $10 \%$ of the time, resulting in a final measure of 45 items. Administration time was approximately $30-40 \mathrm{~min}$.

\section{Data Analyses}

The internal consistency reliability of the APCP was examined through an item analysis of the intensity scale for each of the four activity domains, i.e., play ( 9 items), skill development (15 items), active physical recreation (10 items), and social (11 items) activities. Cronbach's alpha values were reported; values greater than 0.7 were considered acceptable internal consistency (Portney \& Watkins, 2000).

In order to examine whether the APCP can distinguish between subgroups, the sample of 120 children was divided into groups by age, sex, family income, and GMFCS levels. Two age-related subgroups were created by dividing the sample into groups of children based on whether they were under or over the age of 4 years, based on developmental theory (Piaget \& Inhelder, 1964), in which the preoperational stage (age 2 to 6 ) is divided into two sub-stages, and the age of transition to full-time academic-based schooling). Based on these criteria, $45 \%$ of the children were under 4 years of age and $55 \%$ were 4 years old and above. Children were then assigned to subgroups based on GMFCS levels to examine whether the APCP differentiated children based on GMFCS level. As in research conducted by Majnemer et al. (2008) and Palisano et al. (2009), the five GMFCS levels were collapsed to form two groups: children classified in GMFCS levels I-III (62\%) and children classified in GMFCS levels IV-V (38\%). Annual family income, which did not vary significantly by province, was measured by classifying family income as $\$ 60,000$ or above, based on the median income in the 2006 Canadian census (Statistics Canada, 2006). Based on this classification, $43 \%$ of the families had annual incomes lower than $\$ 60,000$, while $57 \%$ reported above median incomes.

Independent $t$-tests were performed to test the differences among the subgroups (i.e., age, sex, income, and GMFCS), and consequently examine construct validity of the APCP. In order to test the magnitude of the differences, effect size values were calculated based on the omega square $\left(\omega^{2}\right)$ formula: $\omega^{2}=\left(t^{2}-1\right) /\left(t^{2}+N_{1}+\right.$ $\left.N_{2}-1\right)^{1}$. These values were interpreted according to Kirk's classification (1996), where $\omega^{2}=0.01$ was considered as a small, $\omega^{2}=0.059$ as a medium, and $\omega^{2}=0.138$ as a large effect size. Analysis of covariance was performed to verify that the differences in the GMFCS are still observed while controlling for sex, age, and income. Finally, to examine the association between number of additional health conditions and levels of participation, and to test the association between the APCP and PEDI scores, Pearson correlations were performed. The magnitude of correlation coefficients was considered moderate if $>0.40$ and strong if $>0.60$ (Domholdt, 2000). The level of significance (two-tailed) was set to 0.01 because of the number of analyses conducted. SPSS 19 was used to conduct all statistical analyses.

\footnotetext{
${ }^{1} t=t$-statistic; $N_{1}=$ number of participants in group $1 ; N_{2}=$ number of participants in group 2.
} 
TABLE 2. Internal Consistency of Items in Each Activity Type

\begin{tabular}{lccc}
\hline & Number of Children & Number of Items & Cronbach's Alpha \\
\hline Participation intensity & & & \\
$\quad$ Play & 118 & 9 & 0.65 \\
Skill development & 113 & 15 & 0.70 \\
Active physical recreation & 89 & 10 & 0.52 \\
Social & 116 & 11 & 0.66 \\
Participation diversity & & & \\
Play & 118 & 9 & 0.76 \\
Skill development & 115 & 15 & 0.85 \\
Active physical recreation & 119 & 10 & 0.76 \\
Social & 120 & 11 & 0.73 \\
\hline
\end{tabular}

\section{RESULTS}

\section{Internal Consistency}

The Cronbach's alpha coefficient for the four domains of the APCP varied from 0.73 to 0.85 for diversity scores and from 0.52 to 0.70 for intensity scores (see Table 2 ). Since the Cronbach's test uses list-wise deletion for missing values, only 89 children were included in this analysis for the Active Physical Recreation scale, as the remainder did not take part in these activities at all, so did not have a diversity or intensity score.

\section{Construct Validity}

As shown in Table 3, significant differences in participation intensity and diversity were found between children under 4 years of age $(N=54)$ and children above 4 years of age $(N=66)$ across all activity types with the exception of diversity and intensity of active physical recreation activities and intensity of play activities. Children aged 4 years and older participated in a greater variety of activities (i.e., diversity) and more frequently (i.e., intensity) than younger children (under 4 years of age). Effect size values were medium $\left(\omega^{2}=0.07\right)$ for participation diversity in skill development, social and total activities, and small for diversity of play activities $\left(\omega^{2}=0.02\right)$. We further explored whether specific items changed by age and found that one item, team sports, was reported in the older group and children with the GMFCS levels < III.

Significant differences in participation diversity and intensity across all activity types were found between children classified in the GMFCS levels I-III compared with children in levels IV-V. Children in levels I-III or less participated in a greater range of activities and more frequently than children in levels IV-V. Effect size values, calculated by $\omega^{2}$, were large and ranged from 0.21 to 0.23 with the exception of overall diversity and intensity of social activities, in which effect size values were medium to large $\left(\omega^{2}=0.08-0.16\right)$. Analyses of covariance accounting for child age, sex, and family income indicated similar differences $(8.3<\mathrm{F}<53.5, p<.005)$ with similar effect size ( 0.07 to 0.33 ) in participation patterns between GMFCS groups.

Significant differences in participation diversity were found between income levels across all activity types. Income-related differences were significant for the intensity of play and skill development activities, but not for intensity of social 


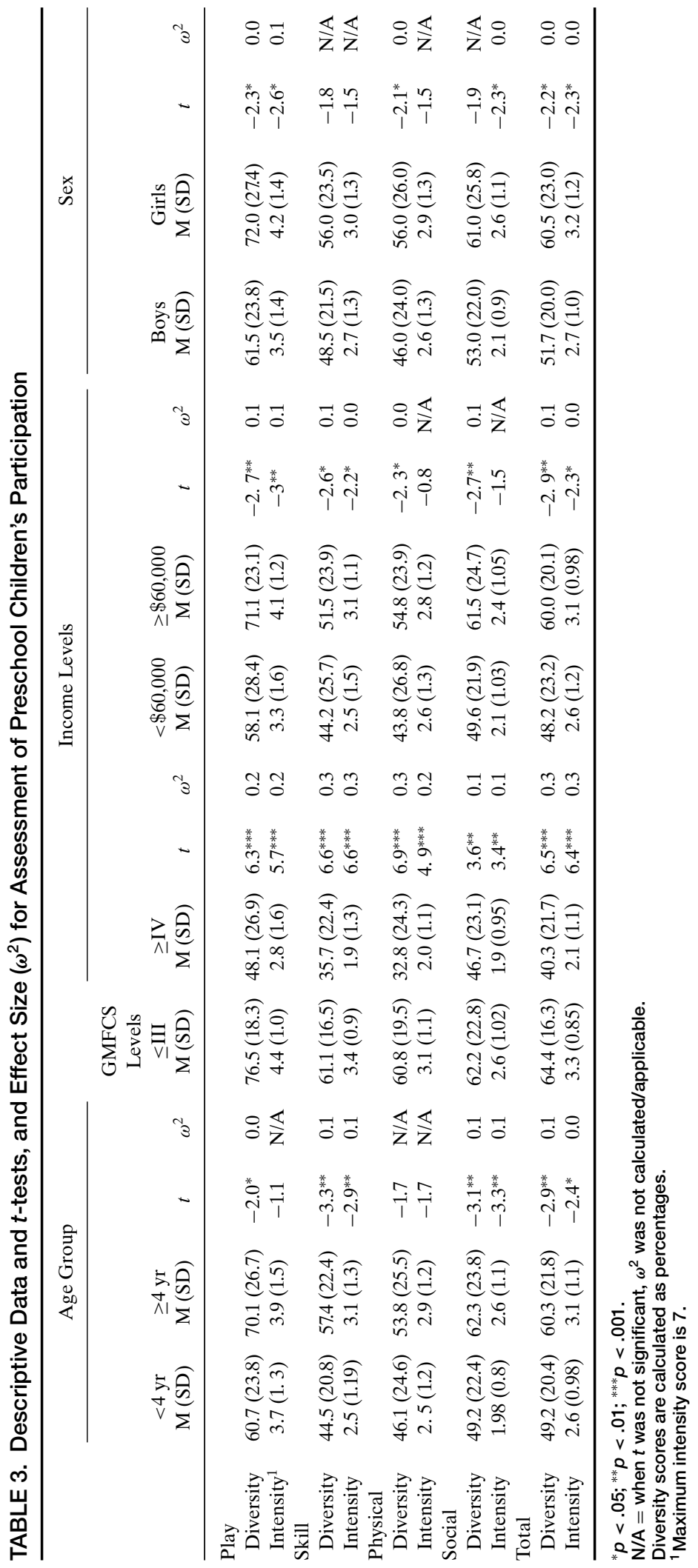


TABLE 4. Pearson Correlations Between the Number of Additional Health Conditions and Assessment of Preschool Children's Participation Scores

\begin{tabular}{|c|c|c|c|c|c|c|c|c|c|c|}
\hline & \multicolumn{2}{|c|}{ Play } & \multicolumn{2}{|c|}{$\begin{array}{c}\text { Skill } \\
\text { Development }\end{array}$} & \multicolumn{2}{|c|}{$\begin{array}{l}\text { Active Physical } \\
\text { Recreation }\end{array}$} & \multicolumn{2}{|c|}{ Social } & \multicolumn{2}{|c|}{ Total } \\
\hline & $N$ & $r$ & $N$ & $r$ & $N$ & $r$ & $N$ & $r$ & $N$ & $r$ \\
\hline Diversity & 120 & $-0.27^{*}$ & 120 & $-0.33^{* *}$ & 120 & $-0.38^{* *}$ & 120 & $-0.35^{* *}$ & 120 & $-0.37^{* *}$ \\
\hline Intensity & 119 & $-0.29^{*}$ & 120 & $-0.40^{* *}$ & 115 & $-0.32^{*}$ & 119 & $-0.37^{* *}$ & 119 & $-0.41^{* *}$ \\
\hline
\end{tabular}

${ }^{*} p<.01 ;{ }^{* *} p<.001$.

and active physical recreation activities. Children of families with higher median incomes participated in more activities and more frequently than children of families with lower median income. Effect size values were small to medium for diversity and intensity ( 0.03 to 0.07$)$. When analyses of variance were performed to account for child age and sex, similar differences $(.002<p<.022)$ with similar effect sizes (0.04 to 0.09 ) were observed.

Differences between boys and girls were found in participation diversity and intensity in play and total activities in which girls participated in a broader range of activities and more frequently than boys (effect size ranged from 0.03 to 0.05 ). In addition, girls participated more intensely in social and more diversely in active physical recreation activities (effect size values were low, both 0.03 ).

Significant negative correlations were found between number of additional health conditions and level of participation diversity and intensity across all activity types (see Table 4). In other words, higher the number of additional health conditions, lower the diversity and intensity of a child's participation in everyday activities.

Finally, positive moderate to strong correlations were found between participation diversity and intensity and levels of performance of self-care and mobility (PEDI scores) across all activity types $(0.51<\mathrm{r}<0.78, p<.001)$. Higher the level of functional skills and independence in performing self-care and mobility tasks, higher the levels of participation (see Table 5).

\section{DISCUSSION}

This paper describes initial validation data for use of the APCP with children with cerebral palsy. Results indicate that the internal consistency of the APCP is good to excellent for diversity scores and moderate for the intensity scores. The relatively lower value of Chronbach's alpha for the active physical recreation intensity scale (0.52) may reflect variability in frequency of participation across children with cerebral palsy in the study sample. Notably, internal consistency values for the intensity scores were not expected to be high, as many factors are expected to influence frequency of participation (King et al., 2006a). Further examination of internal consistency in larger samples of children with cerebral palsy and across a range of diagnoses and developmental stages will indicate whether the current structure of the four scales for the assessment is appropriate. 


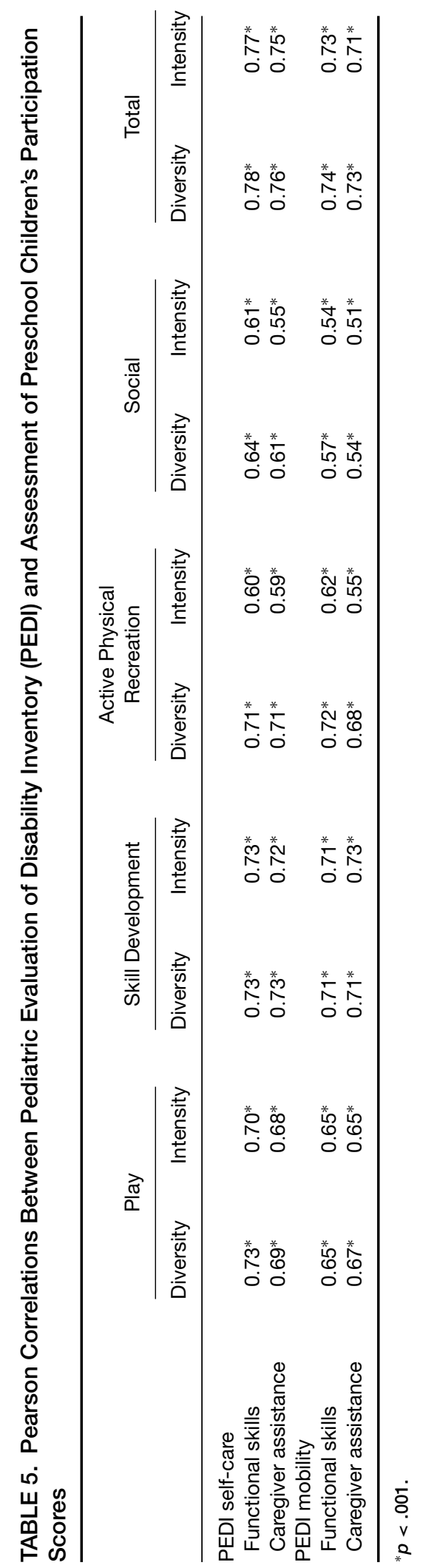


The APCP characterized differences in participation for children with cerebral palsy who were under and over 4 years of age. As hypothesized, when compared with the younger group, children over the age of 4 participated in a broader range of activities and did so with greater intensity. This finding is consistent with developmental theory, which indicates a broadening of interests and activities as preschool children engage in activities outside their home environment more often and begin to be involved in more social play (Piaget \& Inhelder, 1964). Dunst and colleagues (2002) also found that the percentage of children at risk for or with a developmental delay who participated in family and community activities increased significantly from birth to 6 years. Similarly, our findings indicate a higher intensity of participation in skill and social development activities in children with cerebral palsy as their age increases.

Participation profiles of children with disabilities over the age of 6 have found significant differences in both diversity and intensity of participation between boys and girls (Engler-Yeger, 2009; King et al., 2010). Our findings that girls participated more frequently in social activities than boys are similar to the results of studies of children more than 6 years of age (Law et al., 2006). Although the effect size was small, we also found that preschool age girls had more diverse and intense play participation, as well as more diverse active physical participation than boys, contrary to our hypothesis. In our sample, there were more girls with better gross motor functioning (GMFCS I-III) than boys; this might explain the differences in participation levels in these activities. However, no significant correlation was found between sex and GMFCS levels $\left(\chi^{2}=2.8, p=.09\right)$. It is plausible that at this early development stage (age 2 to 6 ), the social implications of the sex of the children do not come into play as much as in later age groups. Also, the greater levels of participation observed in girls may reflect the fact that girls typically develop faster than boys during the preschool years.

Diversity scores of children with cerebral palsy were significantly influenced by levels of family income for all areas; intensity scores were influenced in the activity areas of play and skill activities and overall participation. These findings are similar to other studies completed with children over 6 years of age (Law et al., 2006), which have found income related restrictions in participation in children's everyday activities. This finding highlights the need to make universally accessible early intervention programs and community organizations (e.g., YMCA) available to low-income families.

The construct validity of the APCP is supported by the findings of significant differences, with medium to large effect sizes, across all activity types between children classified in levels I-III and children in levels IV-V on the GMFCS, even after adjustment for the effects of age, sex, and income. Likewise, parents of children with a higher number of health and development conditions reported both lower diversity and intensity of participation across all activity types. Cerebral palsy is a condition characterized by primary motor impairment, and is also associated with many other health and development conditions such as cognitive impairment, communication disorders, epilepsy, and visual and perceptual dysfunction (Dormans \& Pellegrino, 1998). While the finding that GMFCS level and number of health and development conditions are significantly related to participation is not surprising, it does highlight the need to assess levels of participation at an early age. Provision 
of information about participation opportunities and strategies for parents, as well as intervention to modify environments to support participation, can be started at an early age if assessment indicates participation restrictions.

For families and rehabilitation practitioners, the APCP can be used to capture a child's overall level of participation, including the diversity and intensity of activity engagement. Service providers and researchers can use the APCP to assess and monitor children's participation in specific types of activities or patterns of participation, both of which answer basic questions about the nature of preschool children's participation.

These analyses have several limitations. First, the results are from a relatively small sample of children diagnosed with cerebral palsy. Additional studies, including larger samples of children with and without disabilities, will provide additional information about the psychometric properties of this assessment. Likewise, examination of test-retest reliability and confirmation of the factor structure is required.

\section{CONCLUSION}

The APCP is a newly developed measure designed to capture the participation of children aged 2 to 5 years and 11 months in the areas of play, skill development, active physical recreation, and social activities. In this study involving 120 children with cerebral palsy, evidence of internal consistency and the ability to distinguish groups of children with cerebral palsy based on a child's age, sex, GMFCS levels, and level of family income. The APCP, with focus on preschool children, has potential use for assessment and identification of activity areas in which the child is participating and areas in which participation may be restricted.

\section{ACKNOWLEDGMENTS}

Our sincere thanks to the families, children, and therapists who participated in this study. Trial registration number: R01HD044444. This study was funded by the National Institutes of Health, USA. We appreciate the research coordination work provided by Mary Forhan in the development of this measure. Mary Law holds the John and Margaret Lillie Chair in Childhood Disability Research.

Declaration of interest: The authors report no conflicts of interest.

\section{ABOUT THE AUTHOR}

Dr Mary Law, PhD, is affiliated with CanChild Centre for Childhood Disability Research and School of Rehabilitation Science, McMaster University, Hamilton, Ontario, Canada. Dr Gillian King, PhD, is affiliated with Bloorview Research Institute, Toronto, Ontario, Canada. Dr Theresa Petrenchik, PhD, is affiliated with CanChild Centre for Childhood Disability Research and School of Rehabilitation Science, McMaster University, Hamilton, Ontario, Canada. Dr Marilyn Kertoy, PhD, is affiliated with School of Communication Sciences and Disorders, University of Western Ontario, London, Ontario, Canada. Dr Dana Anaby, $\mathrm{PhD}$, is affiliated with School of Physical and Occupational Therapy, McGill University, Montreal, Quebec, Canada. 


\section{REFERENCES}

Anderson, E. M., \& Clarke, L. (Eds.). (1982). Disability and adolescence. New York: Methuen.

Ashford, J. B., \& Leroy, C. (Eds.). (2010). Human behavior in the social environment: A multidisciplinary perspective. (4th ed.). Belmont, CA: Brooks/Cole/Cengage Learning.

Bellamy, T., Newton, S., LeBaron, N., \& Horner, R. (1990). Quality of life and lifestyle outcome: A challenge for residential programs. In R. Schalock \& M. Begab (Eds.), Quality of life: Perspectives and issues (pp. 127-137). Washington, DC: American Association on Mental Retardation.

Berg, C., \& LaVesser, P. (2006). The preschool activity card sort. OTJR: Occupation, Participation and Health, 26(4), 143-151.

Blum, R. W., Resnick, M. D., Nelson, R., \& St. Germaine, A. (1991). Family and peer issues among adolescents with spina bifida and cerebral palsy. Pediatrics, 88(22), 280-285.

Brown, M., \& Gordon, W. (1987). Impact of impairment on activity patterns of children. Archives of Physical Medicine and Rehabilitation, 68, 828-832.

Bult, M., Verschuren, O., Gorter, J., Jongmans, M., Piskur, B., \& Ketelaar, M. (2010). Crosscultural validation and psychometric evaluation of the Dutch language version of the Children's Assessment of Participation and Enjoyment (CAPE) in children with and without physical disabilities. Clinical Rehabilitation, 24(9), 843-853.

Bundy, A. (1997). Test of playfulness (TOP). Fort Collins, CO: Colorado State University.

Cadman, D., Boyle, M., Szatmari, P., \& Offord, D. R. (1987). Chronic illness, disability, and mental and social well-being: Findings of the Ontario child health study. Pediatrics, 79, 805-813.

Coatsworth, J. D., Palen, L., \& Sharp, E. H. (2006). Self-defining activities, expressive identity, adolescent wellness. Applied Developmental Science, 10(3), 157-170.

Cohen, J. (1988). Statistical power analysis for the behavioral sciences (2nd ed.). New Jersey: Lawrence Erlbaum.

Coster, W., \& Khetani, M. (2008). Measuring participation of children with disabilities; issues and challenges. Disability and Rehabilitation, 30(8), 639-648.

Diller, L., Fordyce, D., Jacobs, D., \& Brown, M. (1981). Activity pattern indicators. Rehabilitation inidcators. New York: NY University Medical Centre.

Domholdt, E. (2000). Physical therapy research: Principles and applications (vol. 2). Philadelphia, PA: Saunders.

Dormans, J. P., \& Pellegrino, L. (Eds.). (1998). Caring for children with cerebral palsy: A team approach. Baltimore, MD: Paul H. Brookes.

Dunst, C., Hamby, D., Trivette, C., \& Raab, M. (2002). Young children's participation in everyday family and community activity. Psychological Reports, 91(1), 875-897.

Engel-Yeger, B., Jarus, T., Anaby, D., \& Law, M. (2009). Differences in patterns of participation between youths with cerebral palsy and typically developing peers. American Journal of Occupational Therapy January/February 2009, 63(1), 96-104. doi: 10.5014/ajot.63.1.96

Forsyth, R., \& Jarvis, S. (2002). Participation in childhood. Child: Care, Health \& Development, 28(4), 277-279.

Forsyth, R., Colver, A., Alvanides, S., Woolley, M., \& Lowe, M. (2007). Participation of young severely disabled children is influenced by their intrinsic impairments and environment. Developmental Medicine and Child Neurology, 49(5), 345-9.

Fougeyrollas, P., \& Noreau, L. (1997). Life habits (LIFE-H2.1) ICIDH and environmental factors International Network, 9, 20-31.

Granger, C., Brown, S., Griswold, K., Heyer, N., McCabe, M., Msall, M., et al. (1991). WeeFIM (Functional independence measure for children). Buffalo, NY: Centre for Functional Assessment Research, State University of NY.

Haley, S. M., Coster, W. J., Ludlow, L. H., Haltiwanger, J. T., \& Andrellos, P. J. (1992). Pediatric evaluation of disability inventory (PEDI) development, standardization and administration manual. Boston, MA: PEDI Research Group, New England Medical Center Hospitals.

Hammal, D., Jarvis, S., \& Colver, A. Participation of children with cerebral palsy is influenced by where they live. Developmental Medicine \& Child Neurology, 46, 292-298.

Hay, J. A. (1992). Adequacy in and prediction for physical activity in children. Clinical Journal of Sports Medicine, 2, 192-201. 
Henry, A. D. (1998). Development of a measure of adolescent leisure interests. The American Journal of Occupational Therapy, 52, 531-539.

Imms, C., Reilly, S., Carlin, J., \& Dodd, K. (2008). Diversity of participation in children with cerebral palsy. Developmental Medicine \& Child Neurology, 50(5), 363-369.

Jessen, E., Colver, A., Mackie, P., \& Jarvis, S. (2003). Development and validation of a tool to measure the impact of childhood disabilities on the lives of children and their families. Child: Care, Health and Development, 29(1), 21-34.

King, G., Law, M., King, S., Hurley, P., Rosenbaum, P., Hanna, S., Kertoy, M., \& Young, N. (2004). Children's Assessment of Participation and Enjoyment (CAPE) and Preferences for Activities of Children (PAC). Harcourt Assessment, San Antonio, TX, USA.

King, G., Law, M., Hanna, S., King, S., Hurley, P., Rosenbaum, P., et al. (2006a). Predictors of the leisure and recreation participation of children with physical disabilities: A structural equation modeling analysis. Children's Health Care, 35(3), 209-234.

King, G. A., Law, M., King, S., Hurley, P., Hanna, S., Kertoy, M., et al. (2006b). Measuring children's participation in recreation and leisure activities: Construct validation of the CAPE and PAC. Child: Care, Health and Development, 33(1), 28-39.

King, G., Law, M., Hurley, P., Petrenchik, T., \& Schwellnus, H. (2010). A developmental comparison of the out-of-school recreation and leisure activity participation of boys and girls with and without physical disabilities. International Journal of Disability, Development and Education, 57(1), 77-107.

King, G., Law, M., Petrenchik, T., \& Kertoy, M. (2006c). Assessment of preschool children's participation (APCP). Hamilton, ON: CanChild Centre for Childhood Disability Research, McMaster University.

Kinney, V. B., \& Coyle, C. P. (1992). Predicting life satisfaction among adults with physical disabilities. Archives of Physical Medicine and Rehabilitation, 73, 863-869.

Kirk, R. E. (1996). Practical significance: A concept whose time has come. Educational and Psychological Measurement, 56(5), 746-59.

Knox, S. (1997). Preschool play scale. St. Louis MO: Mosby.

Larson, R. W. (2000). Toward a psychology of positive youth development. American Psychologist, 55(1), 170-183.

Larson, R. W., \& Verma, S. (1999). How children and adolescents spend time across the world: Work, play and developmental opportunities. Psychological Bulletin, 125(6), 701-736.

LaVesser, P., \& Berg, C. (2011). Participation patterns in preschool children with an autism spectrum disorder. OTJR: Occupation, Participation and Health, 31(1), 33-39.

Law, M. (2002). Participation in the occupations of everyday life. The American Journal of Occupational Therapy, 56(6), 640-649.

Law, M., \& Dunn, W. (1993). Perspectives on understanding and changing the environments of children with disabilities. Physical and Occupational Therapy in Pediatrics, 13(3), 1-17.

Law, M., Darrah, J., Pollock, N., Wilson, B., Russell, D. J., Walter, S. D., et al. (2011). Focus on Function: An RCT comparing child versus context-focused intervention for young children with cerebral palsy. Developmental Medicine and Child Neurology, 53(7), 621-629.

Law, M., King, G., King, S., Kertoy, M., Hurley, P., Rosenbaum, P., et al. (2006). Patterns of participation in recreational and leisure activities among children with complex physical disabilities. Developmental Medicine and Child Neurology, 48, 337-342.

Mahoney, J. L., Larson, R. W., \& Eccles, J. S. (2005). Organized activities as contexts of development: Extracurricular activities, afterschool, and community Programs (pp. 3-22). Mahwah, NJ: Lawrence Erlbaum.

Majnemer, A., Shevell, M., Law, M., Birnbaum, R., Chilingaryan, G., Rosenbaum, P., et al. (2008). Participation and enjoyment of leisure activities in school aged children with cerebral palsy. Developmental Medicine and Child Neurology, 50(10), 751-758.

McCubbin, H., McCubbin, M., \& Thompson, A. (1991). Family time and routines index. Madison WI: University of Wisconsin.

Miller, L. J. (1982). Miller assessment for preschoolers. Littleton, CO: Foundation for Knowledge Development. 
Msall, M., DiGuadio, K., Duffy, L., LaForest, S., Braun, S., \& Granger, C. (1994). WeeFIM: Normative sample of an instrument for tracking functional independence in children. Clinical Pediatrics, 33, 431-438.

Newborg, J., Stock, J. R., Wnek, L., Guidubaldi, J., \& Svinicki, J. (1984). Batelle developmental inventory screening test. Allen, TX: DLM Teaching Resources.

Palisano, R. J., Kang, L. J., Chiarello, L. A., Orlin, M., Oeffeinger, D., \& Maggs, J. (2009). Social and community participation of children and youth with cerebral palsy. Physical Therapy, 89(12), 1304-1314.

Palisano, R. J., Rosenbaum, P. L., Walter, S., Russell, D., Wood, E. \& Galuppi, B. (1997). Development and reliability of a system to classify gross motor function in children with cerebral palsy. Developmental Medicine and Child Neurology, 39, 214-233.

Piaget, J., \& Inhelder, B. (1964). The growth of logic in the child. New York: Harper \& Row.

Portney, L. G., \& Watkins, M. P. (2000). Foundations of clinical research: Applications to practice (2nd ed.). Upper Saddle River, NJ: Prentice Hall Health.

Rosenbaum, P., King, S., Law, M., King, G., \& Evans, J. (1998). Family-centred service: A conceptual framework and research review. Physical and Occupational Therapy in Pediatrics, 18(1), $1-20$.

Russell, D. J., Rosenbaum, P. L., Avery, L., \& Lane, M. (2002). Gross motor function measure (GMFM-66 and GMFM 88) user's manual: Clinics in developmental medicine. Holborn, London: MacKeith Press.

Sandler, I., Ayers, T., Suter, J., \& Twohey-Jacobs, J.. (2004). Investing in children, youth, families and communities: Strengths-based research and policy. American Psychological Association, 380, 31-49.

Simeonsson, R., Leonardi, M., Lollar, D., Bjorck-Akesson, E., Hollenweger, J., \& Martinuzzi, A. (2003). Applying the international classification of functioning, disability and health (ICF) to measure childhood disability. Disability and Rehabilitation, 25(11-12), 602-610.

Sloper, P., Turner, S., Knussen, C., \& Cunningham, C. (1990). Social life of school children with Down's syndrome. Child: Care, Health and Development, 16(4), 235-251.

Sparrow, S. S., Balla, D. A., \& Chicchetti, D. V. (1984). Vineland adaptive behavior scales. Circle Pines, MN: American Guidance Service.

Statistics Canada (2006). 2006 census tables income and earnings. Retrieved April 6, 2011, from http://www12.statcan.ca.libaccess.lib.mcmaster.ca/census-recensement/2006/dp$\mathrm{pd} / \mathrm{hlt} / \mathrm{index}$-eng.cfm

Stoffel, A. \& Berg, C. (2008). Spanish translation and validation of the preschool activity card slot. Physical \& Occupational Therapy in Pediatrics, 28(2), 171-189.

Welsh, B., Jarvis, S., Hammal, D., \& Colver, A. (2006). How might districts identify local barriers to participation for children with cerebral palsy? Public Health, 120(2), 167-75.

Whiting, B. B., \& Edwards, C. (1988). Children of different worlds: The formation of social behavior. Cambridge, MA: Harvard University Press.

World Health Organization (WHO). (2001). The international classification of functioning, disability and health. Geneva, Switzerland: WHO.

Wright, M. O. D., \& Masten, A. S. (2005). Resilience processes in development: Fostering positive adaptation in the context of adversity. In S. Goldstein \& R. Brooks, (Eds.), Handbook of resilience in children (pp. 17-37). New York: Kluwer Academic/Plenum.

Young, N. L. (1997). Activities scale for kids (ASK). Toronto ON: Hospital for Sick Children. 